\title{
Screening for colorectal cancer
}

Harvard Medical School, Boston, MA, USA

Colorectal cancer is the second leading cause of cancer death in the U.S. and common throughout the world. The biologic behavior of this cancer suggests that it can be prevented: most cancer arise from adenomas, which progress over many years from small $(<5 \mathrm{~mm})$ lesions to cancer and can be identified during a precancerous phase. Now there is strong evidence that screening prevents incidence and mortality form colorectal cancer but disagreement about the best strategy.

Four screening methods are available. They differ is the strengthy: of evidence supporting them, cost and acceptability. I) The strongest evidence is for testing for fecal occult blood (FOBT): a guaiac-based test on 2 specimens from 3 consecutive stools yearly, with colonoscopy for patients with positive tests. A randomized controlled trial of this strategy showed a reduct on in mortality of one third and others now in progress are showing comparable results. This method aims mainly at early detection of cancer, not polyps, because polyps infrequently bleed. 2) Sigmoidoscopy has been shown to reduce mortality from colorectal cancer by two thirds in the segment of the colon examined but the evidence, from two case control studies, is not as strong as with randomized trials and only about half of the colon is examined. 3) Finding romoving polyps, by colonoscopy, seems to prevent colorectal cancers from arising. Winawer and colleagues have shown that patients who have had their colon cleared of polyps and remain under surveillance with colonoscopy every 1-3 years have an incidence of cancer of only about 20 percent of similar patients, in other cohorts. 4) Finally, double contrast barium enemas detect large (high-risk) polyps and early cancers about as well as colonoscopy and are less expensive and more acceptable. However, there is no direct evidence that screening with barium enemas prevents cancer incidence or mortality and evidence that barium enema and colonoscopy have comparable sensitivity and specificity is not strong.

Screening usually begins at age 50 years: about $90 \%$ of cancers occur after that age. Patients are reluctant to submit stools or undergo endoscopies but their compliance can be improved. The monetary cost of screening for colorectal cancer appears comparable to screening for other common cancers such as breast. The false positive rate is high; as many as 40 positive FOBT tests must be worked up for every cancer found.

Some patients are known, from history and phisical examination, to be at increased risk, for their age, for colorectal cancer. The main risk factors are specific syndromes (familial adenomatous polyposis, hereditary non-polyposis colon cancer, inflammatory bowel disease) and a family history of colon cancer, especially first degree relatives with onset before age 45 years. There is consensus that patients with these conditions should be screened differently (earlier onset of testing, at shorter intervals and with more powerful testing such as colonoscopy rather than FOBT) but no strong evidence favoring any of these strategies. Genes for the inherited syndromes have been identified and can be used to predict risk within affected kindreds but are not presently useful for general screening.

Most current recommendations include yearly FOBT with or without sigmoidoscopy every 5 years, both after age 50 years, with colonoscopy for patients with positive tests. However, new forms of testing and evidence of effectiveness for existing methods are accumulating rapidly and these recommendations are likely to change. 